\title{
Watch What You Swallow!-A Rare Cause of Not So Rare Entity Presenting to the Emergency Room
}

\author{
Pratik Mukherjee $^{1}$ Daniel Wu Peng ${ }^{1}$ Ashish Chawla ${ }^{1}$
}

Address for correspondence Pratik Mukherjee, MMed, FRCR Department of Diagnostic Radiology, Khoo Teck Puat Hospital, 90 Yishun, Central Singapore 768828 (e-mail: dr.mukherjee.p@gmail.com).

\begin{abstract}
Keywords

- computed tomography

- appendicitis

- fishbone

- foreign body

Foreign-body ingestion is a rare cause for acute appendicitis. The authors report a case of a 48-year-old man who presented with abdominal pain for 3 days. Computed tomography (CT) revealed a foreign body in the appendix with peri-appendicular inflammatory changes. The patient underwent a successful appendectomy with complete recovery.
\end{abstract}

\section{Introduction}

The presence of a foreign body in the appendix causing acute appendicitis is very rare. ${ }^{1}$ To the authors' knowledge, there are few case reports and a couple of short case series about appendicitis secondary to foreign bodies in the literature..$^{2-6}$ In a recent systematic review published in 2017, the authors concluded that globally the incidence of appendicitis has been rising over the past decade, although the incidence of appendectomy has steadily stabilized in nonperforated appendicitis since 1990. On the other hand, the incidence of appendicitis or appendectomy is high in newly industrialized countries in Asia in the 21st century. ${ }^{7}$ Here the authors present a rare case of appendicectomy for acute appendicitis due to an ingested foreign body.

\section{Case Report}

A 48-year-old man came to emergency department presenting with 2 days of abdominal pain. The pain started in a generalized pattern and migrated to the right iliac fossa with gradual increase in intensity. On physical examination, the abdomen was soft and mildly distended. There was no guarding or rigidity. The full blood count showed elevated white cells $\left(13.36 \times 10^{9} / \mathrm{L}\right)$ and neutrophils $\left(9.110^{9} / \mathrm{L}\right)$.

Routine abdominal radiograph showed a faint linear density at the right lower abdomen (-Fig. 1). At this point, the reporting radiologist went back to the emergency room to discuss the case with referring clinician, and additional history of possible accidental ingestion of fishbone 2 days back was provided by the patient. The patient subsequently underwent contrast-enhanced computed tomography (CT) of the abdomen and pelvis, which revealed a curvilinear density measuring approximately $1.8 \mathrm{~cm}$ within the appendix ( - Fig. 2). It appeared to have penetrated the posterior wall of the appendix with a small phlegmon at the suspected site of perforation. These were better demonstrated in the coronal and sagittal reformats. The volume-rendered images were quite useful in demonstrating the shape and appearance of the foreign body ( $\boldsymbol{- \text { Fig. }} \mathbf{3}$ ). The appendix was dilated and thickened with surrounding fat stranding and fluid.

Exploratory laparoscopy of the abdomen confirmed an abnormally dilated appendix and the presence of foreign body penetrating the appendicular wall. The patient underwent appendectomy and retrieval of the foreign body. The foreign body was compatible to a fishbone measuring approximately $2 \mathrm{~cm}$ ( - Fig. 4). The histologic result of the resected appendix showed transmural suppurative inflammation and necrosis, consistent with acute appendicitis. The patient was observed for 2 days postoperatively and subsequently discharged well.

\section{Discussion}

Patients' presentation to the emergency department with right iliac fossa pain is quite common. ${ }^{8}$ Appendicitis is one of the most common causes in the general population with no

\section{received}

October 16, 2018

accepted

October 17, 2018
DOI https://doi.org/

10.1055/s-0038-1676408
(C)2019 Indian Society of

Gastrointestinal and Abdominal Radiology
License terms

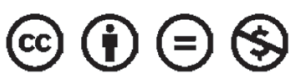


specific age or sexual preference. ${ }^{1}$ Emergency visits related to gastrointestinal foreign bodies are relatively common causes of emergency admission. ${ }^{1,8}$ Generally, the ingested foreign body pass through the gastrointestinal tract without complications. ${ }^{2}$ The incidence of acute appendicitis caused by a foreign body is very rare. ${ }^{3}$

The ingested foreign bodies tend to gravitate and lodge at the cecum due to its dependent position and relatively low motility. The appendiceal orifice can expand and allow occasional entry of a foreign body into its lumen. However,

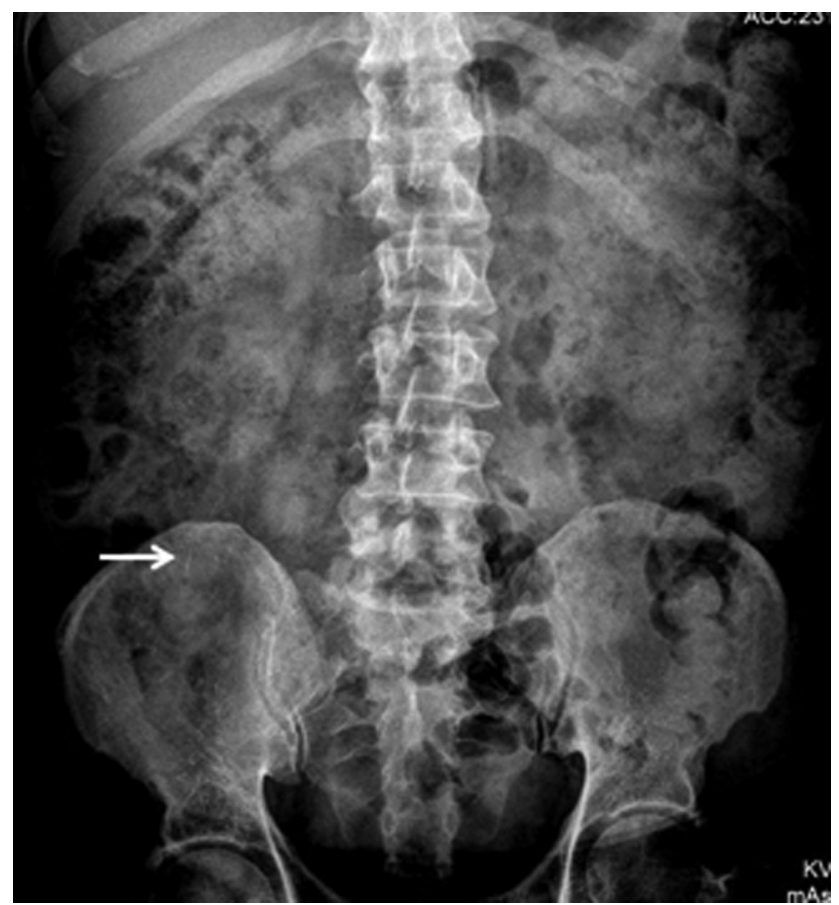

Fig. 1 Abdominal radiograph shows a faint linear density in the right lower abdomen (white arrow). this rare chance of entry also depends on the anatomic position of the appendix. There is almost no possibility of an object entering into a retrocecal appendix. ${ }^{4}$ Various foreign bodies have been reported in the literature as a cause for appendicitis such safety pins, needles, drill bits, seeds, and condom. ${ }^{4-6,9}$ Elongated, sharp foreign bodies are more prone to cause perforation, appendiceal abscess, and peritonitis. ${ }^{4}$

The symptoms vary from being asymptomatic to abdominal pain. Patients may present with low-grade fever, abdominal tenderness on examination, and have elevated inflammatory markers on blood examination. ${ }^{5}$ Proper history taking usually reveals a history of accidental foreign-body ingestion in most patients.

Usually, the first line of investigation in patients with history of foreign-body ingestion presenting to the emergency room is to perform an abdominal radiograph to detect the location of the foreign body and any associated major complications. CT is used for cases in which the cause of the pain is not found, or for confirming the diagnosis and evaluating for complications. ${ }^{1,8,10}$ Diagnostic laparoscopy has been described as feasible for foreign bodies in the gastrointestinal tract including the appendix. ${ }^{2}$

\section{Conclusion}

Acute appendicitis secondary to ingested foreign body is a rare phenomenon. The reporting radiologist should be aware of the appearances of various foreign bodies in the abdomen and should have a high clinical suspicion in patients with history of foreign-body ingestion presenting with right lower quadrant pain. The cross-sectional imaging should be reviewed on multiple planes to avoid dismissing the foreign body as an appendicolith and also look for secondary complications such as perforation and abscess formation that may be more evident on coronal or sagittal planes as in this case.

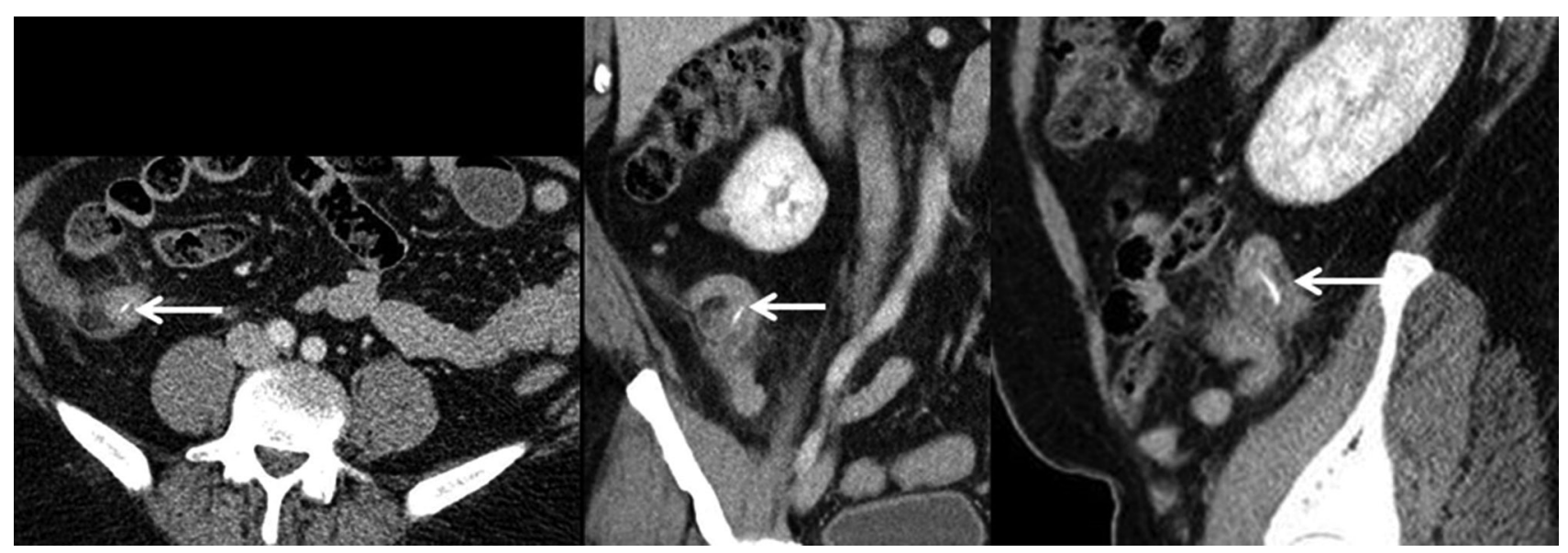

Fig. 2 Axial, coronal, and sagittal CECT (contrast enhanced computed tomography) images in the portal-venous phase show a dilated and thickened appendix with surrounding fat stranding and fluid, with curvilinear density (white arrow) within it. This appears to have penetrated the posterior wall (best appreciated on the sagittal image), and is consistent with the patient's history of fishbone ingestion. 


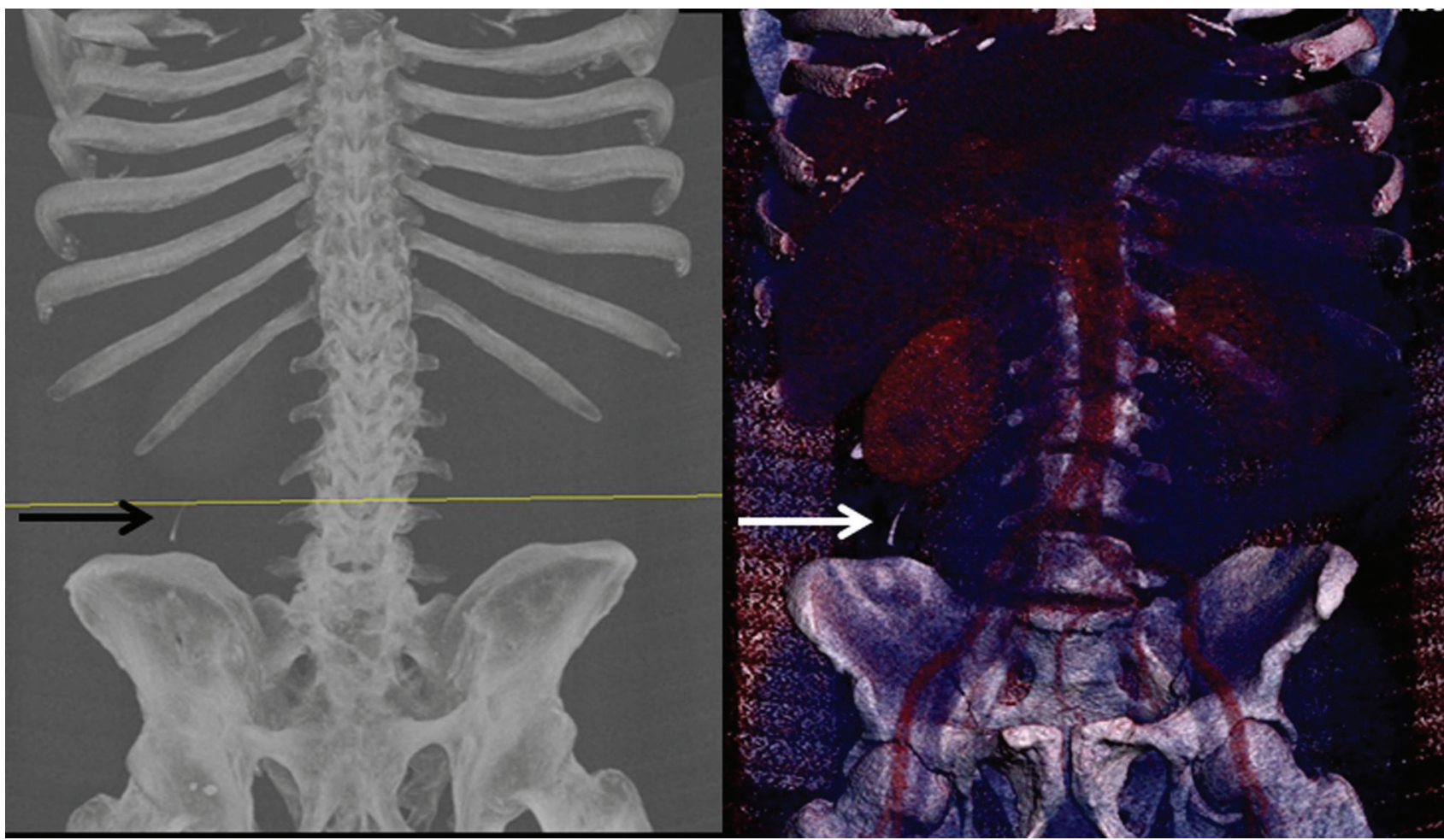

Fig. 3 Coronal images of $\mathrm{CT}$ of the abdomen and pelvis with maximum intensity projection (MIP) reconstruction (left) and volume-rendered image demonstrate the ingested foreign body.

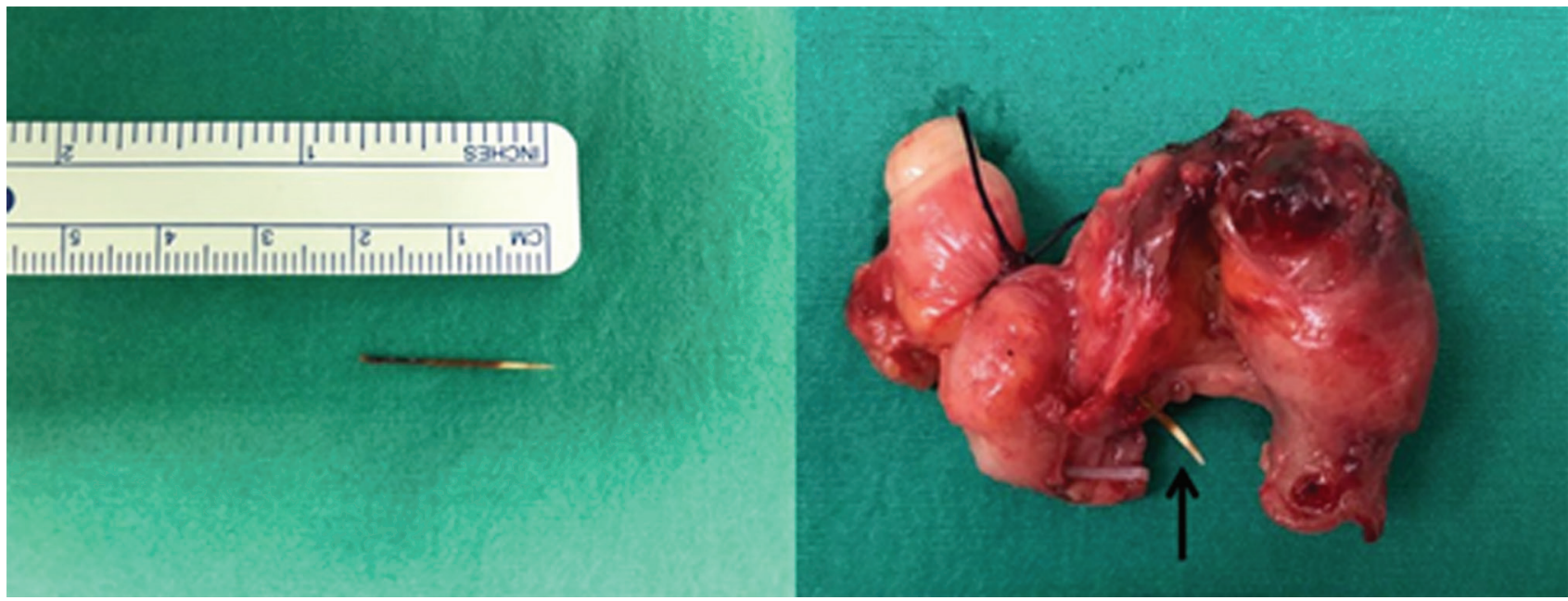

Fig. 4 Foreign body retrieved from appendiceal lumen (left) and resected appendix (right). In the right-hand specimen image, the foreign body can be seen protruding out (black arrow).

\section{Authors' Contributions}

Pratik Mukherjee reported the case and came up with the idea of writing up. Daniel Wu Peng wrote up the case together with the author Pratik Mukherjee. Ashish Chawla reviewed the report and gave valuable inputs in the write-up.

\section{Conflict of Interest}

None declared.

\section{References}

1 Lee JH, Kim HC, Yang DM, et al. What is the role of plain radiography in patients with foreign bodies in the gastrointestinal tract? Clin Imaging 2012;36(5):447-454

2 Klingler PJ, Seelig MH, DeVault KR, et al. Ingested foreign bodies within the appendix: a 100-year review of the literature. Dig Dis 1998;16(5):308-314

3 Balch CM, Silver D. Foreign bodies in the appendix. Report of eight cases and review of the literature. Arch Surg 1971;102(1):14-20 
4 Benizri EI, Cohen C, Bereder JM, Rahili A, Benchimol D. Swallowing a safety pin: report of a case. World J Gastrointest Surg 2012;4(1):20-22

5 Hazer B, Dandin O, Karakaş DO. A rare cause of acute appendicitis: an ingested foreign body. Ulus Travma Acil Cerrahi Derg 2013;19(6):570-572

6 Song YS, Covarrubias DA, Nardi PM. Foreign body appendicitis. AJR Am J Roentgenol 2009;193(2):W154-5

7 Ferris M, Quan S, Kaplan BS, et al. The global incidence of appendicitis: a systematic review of population-based studies. Ann Surg 2017;266(2):237-241

8 Katsinelos P, Kountouras J, Paroutoglou G, Zavos C, Mimidis K, Chatzimavroudis G. Endoscopic techniques and management of foreign body ingestion and food bolus impaction in the upper gastrointestinal tract: a retrospective analysis of 139 cases. J Clin Gastroenterol 2006;40(9):784-789

9 Sama CB, Aminde LN, Njim TN, Angwafo FF 3rd. Foreign body in the appendix presenting as acute appendicitis: a case report. J Med Case Reports 2016;10:129

10 Sar S, Mahawar K, Marsh R, Small PK. Recurrent appendicitis following successful conservative management of an appendicular mass in association with a foreign body: a case report. Cases J 2009;2:7776 